\title{
Deep Venous Thrombosis in an Amputated Limb Stump of a Diabetic Patient: A Case Report
}

\author{
Taoreed A.Azeez, MBChB, MSc, MWACP ${ }^{\prime *}$;Arionola Esan, MBBS, FWACP'; Taiwo R. Kotila, MBBS, FMCPath ${ }^{2}$ \\ 'Endocrinology Unit, University College Hospital, Ibadan, Nigeria \\ ${ }^{2}$ Haematology Department, University College Hospital, Ibadan, Nigeria \\ "Corresponding author \\ Taoreed A. Azeez, MBChB, MSc, MWACP \\ Senior Registrar, Endocrinology, Metabolism and Diabetes Unit, Department of Medicine, University College Hospital, Ibadan, Nigeria; \\ E-mail: adegokegalaxy@yahoo.com
}

\section{Article information}

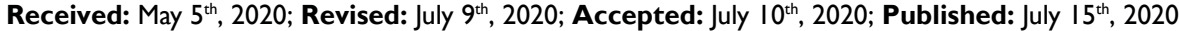

\section{Cite this article}

Azeez TA, Esan Arionola, Kotila TR. Deep venous thrombosis in an amputated limb stump of a diabetic patient:A case report. Diabetes Res Open J. 2020; 6(I): 17-19. doi: 10.17|40/DROJ-6-145

\section{ABSTRACT}

Deep venous thrombosis (DVT) in an amputated stump is potentially life-threatening but rarely diagnosed and there are limited data in sub-Saharan Africa. This is aimed at demonstrating an additional vascular risk in patients with lower limb amputation and diabetes. A 74-year-old man who had a right above knee amputation done on account of grade 5 right diabetic foot with post-operative prophylactic anticoagulation. Doppler ultrasound done before the surgery showed bilateral multiple lower limb arteries atherosclerosis but no evidence of deep venous thrombosis. He was discharged home on Zimmer frame. Three months after, he was noticed to have differential swelling of the right amputation stump. Thigh circumference measured at $15 \mathrm{~cm}$ below the anterior superior iliac spine was $55 \mathrm{~cm}$ and $50 \mathrm{~cm}$ on the right and left respectively there was but no differential warmth or tenderness. The vital signs were relatively stable. Doppler ultrasound scan of the lower limbs showed an echogenic thrombus in the right deep femoral vein. He was commenced on therapeutic dose of subcutaneous enoxaparin. DVT in an amputated stump is uncommonly encountered. It may not have classical clinical findings. Poor mobility and pooling of venous blood in the amputated stump are some of the risk factors that have been reported. DVT in an amputated stump in a patient with diabetes is rare and may not present classically. It may be one of the potential reasons for the increased mortality after lower limb amputation.

\section{Keywords}

Deep venous thrombosis (DVT); Amputated stump; Diabetes patient.

\section{INTRODUCTION}

$\mathrm{D}$ iabetes mellitus is the commonest reason for non-traumatic amputation. ${ }^{1}$ Deep venous thrombosis (DVT) is the formation of blood clot in a deep vein. DVT affects the lower limbs most commonly. Major amputation procedure in a patient with diabetes is associated with an increased risk of deep venous thrombosis in the immediate post-operative period. ${ }^{2}$ DVT complicating an amputated limb in patients with diabetes is not a commonly reported phenomenon after the immediate post-operative period. The incidence of DVT in amputated stumps is so rare that the initial redness and swelling may be considered as features of an infectious process like cellulitis. ${ }^{3}$ The classical risk factors are represented by the Virchow's triads-stasis, hypercoagulability and damaged endo- thelium. ${ }^{4}$ DVT in the amputation stump is not frequently encountered and there is limited data in sub-Saharan Africa.

The objective of this case report is to demonstrate that DVT in an amputated stump is an additional vascular risk in patients with lower limb amputation and diabetes.

\section{CASE PRESENTATION}

The patient is a 74-year-old man who was diagnosed with type 2 diabetes mellitus (T2DM) 20-years earlier. He was not adherent with his oral glucose lowering agents (Metformin and glimepiride). The short-term and long-term glycemic control were suboptimal. 
He presented with a right foot ulcer. He was walking in his house barefooted and he had a small nail puncture injury to the right sole. He did not feel pain but he noticed the bleeding.

The ulcer progressed within a few days, got swollen and started discharging pus. The ulcer later extended to involve the whole of the right sole and the distal part of the dorsum. The tissue turned darkish, involving the whole right foot, with foulsmelling purulent discharge. There was associated high grade fever, some episodes of postprandial vomiting, reduced appetite and generalized body weakness. There was no loss of consciousness.

The essential findings on general physical examination were pallor, fever $\left(38.4^{\circ} \mathrm{C}\right)$ and moderate dehydration. Cardiovascular examinations showed tachycardia (pulse rate of 106 beats per minute) and elevated systolic blood pressure $(164 / 80 \mathrm{mmHg})$. The left foot showed some clawing of toes, dystrophic nails and loss of protective sensation using $10 \mathrm{~g}$-monofilament testing. Dorsalis pedis artery and posterior tibial artery pulsations were felt. The right foot showed an extensive ulcer involving the whole sole and the dorsum. It was covered with necrotic tissue with foul-smelling discharge. There was ascending fasciitis up to the right knee.

The assessment was Meggitt-Wagner grade 5 right diabetic foot and sepsis. Among other investigations done, doppler ultrasound before the surgery showed bilateral multiple lower limb arteries atherosclerosis. There was no evidence of deep venous thrombosis prior to the surgery.

He eventually had a right above knee amputation done on account of grade 5 right diabetic foot and he had post-operative prophylactic anticoagulation. The recovery was uneventful and he was discharged home on Zimmer frame. He was seen twice at the Endocrinology and Orthopedic Clinics and there was no remarkable development. The healing was satisfactory and glycemic control had improved. The pre-operative HbA1c was 11.4 while the HbA1c 3-months after was $8.6 \%$.

Three months later, he presented with differential swelling of the right amputation stump. He had first noticed it 2-days prior to presentation. There were no respiratory symptoms, chest pain or syncopal attack. Thigh circumference measured at $15 \mathrm{~cm}$ below the anterior superior iliac spine was $55 \mathrm{~cm}$ and $50 \mathrm{~cm}$ on the right and left respectively but there was no differential warmth or tenderness. The vital signs were relatively stable. A clinical suspicion of deep venous thrombosis involving the amputated stump was entertained. Doppler ultrasound scan of the lower limbs showed an echogenic thrombus in the right deep femoral vein. $\mathrm{He}$ was commenced on therapeutic dose of subcutaneous enoxaparin. He was later changed to Warfarin and he was followed-up at the Hematology Clinic. There were no further complications.

\section{DISCUSSION}

Foot ulceration and amputation which in the presence of common predisposing factors such as peripheral neuropathy, peripheral arterial disease and poor glycemic control, is a common cause of morbidity and mortality in people with diabetes. ${ }^{5}$ Despite the presence of risk factors, uncertainty still exists about the incidence of DVT after lower extremity amputation. ${ }^{6}$ However, an incidence rate of about $50 \%$ has been reported in the literature. ${ }^{4}$ It has also been found that DVT in an amputated stump is more common following above knee amputation, as found in this case report also. ${ }^{4}$ Classical symptoms and signs of DVT are often absent which may lead to an underestimation of the problem. ${ }^{7}$ Therefore further examination of the patients with duplex scanning is often required to ascertain the diagnosis. Suggested explanations for DVT in an amputated stump include immobility and increased venous pooling of blood in the amputated limb as a result of loss of soleal pump that assists venous drainage of the lower limbs. ${ }^{6}$

\section{CONCLUSION}

Deep venous thrombosis in an amputated stump in a patient with diabetes may not present with classical symptoms and signs and a high index of suspicion is needed to make a diagnosis. It may be also account for the well-documented increased cardiovascular mortality after lower limb amputation in patients with diabetes.

\section{CONSENT}

The authors have received written informed consent from the patient.

\section{CONFLICTS OF INTEREST}

The authors declare that they have no conflicts of interest.

\section{REFERENCES}

1. Bharara M, Mills JL, Suresh K, Rilo HL, Armstrong DG. Diabetes and landmine-related amputations: A call to arms to save limbs. Int Wound J. 2009; 6(1): 2-3. doi: 10.1111/j.1742481X.2009.00587.x

2. Matielo MF, Presti C, Casella IB, Netto BM, Puech-Leão P. Incidence of ipsilateral postoperative deep venous thrombosis in the amputated lower extremity of patients with peripheral obstructive arterial disease. J Vasc Surg. 2008; 48(6): 1514-1519. doi: 10.1016/j.jvs.2008.07.055

3. Chong DK, Panju A. Deep venous thrombosis as a cause of stump swelling in two lower extremity amputee patients. Arch Phys Med Rebabil. 1993; 74: 1002-1003.

4. Buke B, Kumar RR, Vickers V, Grant E, Scremin E. Deep venous thrombosis after lower limb amputation. Am J Phys Med Rehab. 2000; 79 (2): 145-149. doi: 10.1097/00002060-20000300000006

5. American Diabetes Association. Microvascular complications and foot care: Standards of mdical care in diabetes-2020. Diabetes Care. 2020; 43(Supplement 1): S135-S151. doi: 10.2337/dc20S011 
6. Pinar MP, Toledo RP, Castro AH. Deep venous thrombosis (DVT) in lower extremity amputation as a cause of pulmonary embolism. EJCRIM. 2015; 2. doi: 10.12890/2015_000218

7. Matielo MF, Presti C, Casella IB, Puech-Leao P. Incidence of ipsilateral post-operative deep vein thrombosis in the amputated lower extremity of patients with peripheral obstructive arterial disease. J Vasc Surg. 2008; 48: 1514-1519. doi: 10.1016/j. jvs.2008.07.055 